\title{
USEFUL ABBREVIATIONS
}

BANA British, Australasian, North American (Holliday 1995)

BNC British National Corpus

BAWE British Academic Writing in English Corpus

CANCODE Cambridge-Nottingham Corpus of Discourse in English

CLIL content and language integrated learning

CLL Community language learning (Curran 1983)

CLT Communicative language teaching

EAP English for Academic Purposes

EIL English as an International Language

ELF English as a Lingua Franca

ELT English Language Teaching

ENL English as a Native Language

ESP English for Specific Purposes

HOT Higher order thinking

ICT Information communication technology

LAB Language Aptitude Battery (Pimsleur 1966)

LAD Language Acquisition Device (Chomsky 1965)

LL language learning

LT language teaching

MLAT Modern Languages Aptitude Test (Carroll and Sapon 1955)

PPP Presentation Practice Performance

SLA Second Language Acquisition

TBL Task-based learning (Ellis 2003; Eckerth and Siekman 2008)

TESEP Tertiary, Secondary and Primary (Holliday 1995)

TPR Total Physical Response (Asher, Kusudo and de la Torre 1983) 